\title{
XANTHINE BASES IN SUGAR-CANE.
}

BY EDMUND C. SHOREY.

Received April 3, 1899.

F a sample of cane-juice be clarified in the ordinary way with lead subacetate, the excess of lead removed by sodium carbonate, or hydrogen sulphide, the solution made alkaline with sodium hydroxide, if not already so, a few drops of Fehling's solution added, and heated to boiling, a whitish precipitate is formed which rapidly becomes green. This was thought to indicate the presence of xanthine bodies. When solutions containing xanthine bases and reducing bodies are treated in this way with Fehling's solution, cuprous oxide is first formed by the action of the reducing bodies, and with this cuprous oxide the xanthine bases unite forming a greenish-white precipitate, $\mathrm{Cu}_{2}$ combining with one molecule of the xanthine body. If the Fehling's solution be in excess, and there be also an excess of reducing bodies present, there is of course an excess of cuprous oxide formed, the red color of which hides that of the xanthine compound.

In order to isolate the suspected xanthine base, about ten liters of cane-juice were treated in the way outlined above, enough Fehling's solution being added to give a decidedly red precipitate, the solution filtered, the precipitate well washed, and dissolved in dilute nitric acid. To this solution ammoniacal silver nitrate was added, when a dirty brown precipitate was thrown down.

This silver nitrate precipitate was found to dissolve in nitric acid (sp. gr. I. Io) somewhat slowly on boiling, and the solution so obtained deposited crystals slowly. This indicated the presence of guanine and possibly also of xanthine. A portion of the silver nitrate precipitate was treated with dilute hydrochloric acid, filtered from silver chloride, and the solution evaporated, when crystals were formed having the characteristic microscopic appearance of guanine hydrochloride. The crystals were all of one form, and none of the characteristic crystals of xanthine hydrochloride were observed, proving that no other xanthine body than guanine was present in any quantity. 
The guanine hydrochloride so obtained, when treated in water solution, with an excess of ammonia yielded a flocculent white precipitate of guanine, insoluble in water, alcohol, ether, and hot dilute ammonia. This guanine was further identified by Strecker's test: evaporation to dryness with nitric acid and treatment of the residue with caustic potash, when on heating a yellowish red coloration is obtained; also by its conversion into xanthine by nitrous acid:

$$
\mathrm{C}_{6} \mathrm{H}_{6} \mathrm{~N}_{6} \mathrm{O}+\mathrm{HNO}_{2}=\mathrm{C}_{5} \mathrm{H}_{4} \mathrm{~N}_{4} \mathrm{O}_{2}+\mathrm{N}_{2}+\mathrm{H}_{2} \mathrm{O} \text {, }
$$

and the formation of the characteristic crystals of xanthine hydrochloride on treatment with hydrochloric acid.

This isolation and identification of guanine has been repeated on ten samples of juice from mature cane, and on four samples of refuse molasses, and in each case there has been no indication of the presence of any other xanthine body than guanine. It is reasonable then to conclude that in mature sugar-cane guanine is the predominant and probably the only xanthine body present.

During the present season an attempt has been made to estimate the amount of guanine in cane-juice, operating in the following manner: The juice was clarified with lead subacetate, the excess of lead removed by sodium carbonate, and to the filtrate from lead carbonate, heated to boiling, enough Fehling's solution was added to give a decidedly red precipitate; the solution was filtered, and the precipitate well washed with hot water, and the nitrogen contained therein determined and calculated to guanine. One hundred grams of juice were used for each determination and the nitrogen determined by the Gunning method. An average of six samples of juice of approximately the same purity and composition gave

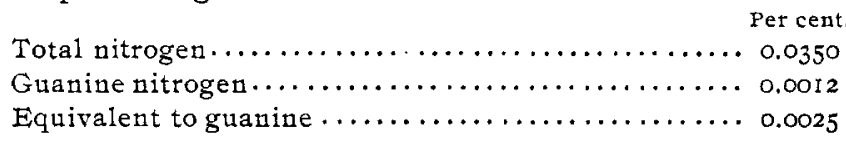

From the known properties of guanine, it was thought that this body would go through the processes of sugar manufacture unchanged, but that inasmuch as some of the other nitrogenous bodies are removed during manufacture, the ratio of guanine nitrogen to total nitrogen would be higher in the refuse molasses 
than in the original juice. This conjecture was confirmed by guanine determinations in molasses. The average of four samples of refuse molasses of similar composition gave

Total nitrogen .............................. 0.7140

Guanine nitrogen............................ 0.0308

Equivalent to guavine $. . . \ldots \ldots \ldots \ldots \ldots \ldots \ldots \ldots . . .0660$

In the samples of raw juice examined the ratio of total nitrogen to guanine nitrogen is $100: 3.42$, while in the refuse molasses the ratio is $100: 4.31$.

The small amount of guanine present in cane-juice is of little technical importance. The amount present in refuse molasses may however be worthy of note in considering the fertilizing or feeding value of the same.

In the analysis of sugar-cane and its products the presence of guanine may introduce an error in some cases unless its presence be recognized. Three of these cases it may be well to note :

I. In the estimation of reducing sugars in technical work the volumetric method with Fehling's solution is generally used; but sometimes when greater accuracy is wished the gravimetric method is used. Now we have already noted that xanthine bodies if present unite with the cuprous oxide formed, and while in the volumetric method this fact introduces no error-for the amount of copper precipitated is not affected-in the gravimetric method by which the cuprous oxide is reduced in a stream of hydrogen, the error introduced is proportional to the amount of guanine or other xanthine base present. The guanine is not affected by the reducing process, and is weighed as copper. It is recommended then that when a gravimetric estimation of reducing sugars is to be made in low grade sugar-house products the electrolytic estimation of the copper be resorted to.

2. A solution of sodium phosphotungstate in sulphuric acid is commonly used as a precipitant of nitrogenous bodies. Guanine in solution is partially precipitated by this reagent not as a compound of phosphotungstic acid but with the sulphuric acid, this compound being sparingly soluble. This may lead to the nitrogen of guanine being credited to other bodies; e. $g$., peptones. A preliminary treatment with sulphuric acid and filtering before addition of sodium phosphotungstate will remove this source of error. 
3. When cane-juice has been clarified with lead subacetate and mercuric nitrate added to the clear solution, a white precipitate is thrown down. This precipitate contains any amide bodies present and by some chemists this use of mercuric nitrate has been proposed or used as a proof of the presence of amide bodies. This is altogether unwarranted, for the mercuric nitrate precipitate contains any guanine present, so that the formation of a precipitate in this case is not only no indication of the amount of amide present, but no evidence of their presence at all.

KöALA, HAWAII,
MatCh $55,1899$.

[CONTRIBUTION FROM THE CHEMICAI LABORATORY OF THE PENNSYLVANIA STAte COLlegF AgRicultural Experiment Station.]

\section{A CONTRIBUTION TO THE CHEMISTRY OF BUTTER-FAT. ${ }^{1}$}

BY C. A, BROWNE, JR.

Received April $1 \%, \mathbf{8 8 9 9 .}$

T

$\mathrm{HE}$ results on the chemistry of butter-fat herewith presented constitute partly the work on regular experiments at the Pennsylvania Experiment Station, and partly extra work performed at odd moments as the other duties of the laboratory permitted.

Nearly all of the analyses were on samples of butter made at the Station Creamery, and representing for the most part the product of a herd of high-grade Guernseys. The figures given in the tables, accompanying this article, need not be taken, therefore, as typical of butter-fats in general, though we believe them to be, on the whole, fairly representative. The differences, if there are any, in the chemical composition of butter-fat from different breeds of cows, is a subject at present being investigated at this station.

We have chosen, for the sake of convenience, to divide our subject into three distinct heads; viz.,

I. The physical and chemical constants of butter-fat.

2. The chemical composition of butter-fat.

3. The chemistry of rancidity in butter-fat.

Each of these divisions will constitute a separate paper.

1 Read by title before the New Yark meeting of the American Chemical Society, December 28, 1898 . 